\title{
Disruptive technology will transform what we think of as robotic surgery in under ten years
}

\author{
Joel Dunning \\ Department of Cardiothoracic Surgery, James Cook University Hospital, Middlesbrough, UK \\ Correspondence to: Joel Dunning. James Cook University Hospital, Marton Road, Middlesbrough TS4 3BW, UK. Email: Joeldunning@doctors.org.uk.
}

\begin{abstract}
What is currently described as 'robotic surgery' will undergo a transformation as rapid as the transformation from Kodak film cameras to digital cameras, from Nokia phones to iPhones, from listening to music on tape to CD then to digital downloading, and the transformation that is shortly to come from petrol cars into electric cars. We are only at the very infancy of what is currently termed robotic surgery, but in reality, is in fact just computer-assisted, instrument-controlled surgery. This article will describe the current state of robotic surgery and will then go on to look at why we will hardly recognize the surgical world in ten years' time, as novel, disruptive technology transforms our specialty.
\end{abstract}

Keywords: Robotic surgery; thoracic surgery; wristed instruments

Submitted Feb 25, 2019. Accepted for publication: Mar 01, 2019.

doi: 10.21037/acs.2019.03.02

View this article at: http://dx.doi.org/10.21037/acs.2019.03.02

In 1910 in New York, the roads were full of horses and carriages. One percent of road transport was mechanized, but by 1923 , only 13 years later, $99 \%$ of registered road transport was using the internal combustion engine. Home televisions were present in $10 \%$ of households in 1950 and $90 \%$ in 1960 , and the same transformational rapid change occurred with the introduction of the microwave, the credit card, the transformation of records to tape, then to CD then MP3, all going from being novel devices to being over $90 \%$ adopted by the population within ten years. When disruptive technology enters a market and is a genuine innovative change in technology, worldwide change occurs rapidly. Kodak, a company with a peak annual profitability of $\$ 12 \mathrm{bn}$, filed for bankruptcy in 2012 after the change to digital photography left it blind-sighted. It is not only true that this change happens, and happens very frequently, but with modern connectivity, the rate of this change is increasing more rapidly, and step changes such as iPads, or the advent of Twitter, Snapchat and Uber, happen within a few years now. So, does this transformational change happen in Thoracic Surgery? The answer is that we have likely seen it already.

Diego Gonzalez Rivas shook the specialty with his highly innovative approach to minimally invasive surgery. One of the most remarkable elements of his innovation was not necessarily the actual creation of uniportal surgery, but the speed and enthusiasm with which he managed to change the face of the specialty. Having only invented and performed the first case of uniportal surgery in 2011, it is now the predominant version of minimally invasive thoracic surgery across Asia and in many parts of the world. Rivas illustrated that change does not have to be slow and does not have to wait for three years, multicentre trials. Combined with passion and online modalities such as YouTube, he showed that we can share ideas and develop new concepts very rapidly with the new era of multimedia sharing of cases, videos and sparks of innovation.

Minimally invasive thoracic surgery was created in 1992, but throughout the 1990s and the early millennium, adoption was slow and hampered by inadequate stapling, vision and the lack of any specialized instruments. However, we have witnessed in the UK a change from $9 \%$ to $50 \%$ in only the last five years, as our instrumentation, mentoring, and specialization has rapidly accelerated. Over the next five years, I believe that this figure for VATS will actually become static and then reverse as the robotic figures increase exponentially from less than $5 \%$ now, to around $50 \%$ by 2023 . But even what we call 'robotics' today will 
not be the robotics of five years' time, as at least eight new robotic systems come onto the market over the next three years. They will miniaturize, they will crash in price, they will bring us back to the patient's bedside, they will overlay imaging, or they will provide safety warnings or enhanced pre-operative planning or the ability to rehearse the operation in advance. They will certainly transform training and bring us into line with the flight simulator model of the airline industry and remove the need for us to train on patients, and instead train on simulators.

It would be unwise to think that patient profiles will stay the same while we develop our instrumentation and techniques, as they will not. Lung cancer screening after trials, such as The NLST Trial and the NELSON trial, demonstrating a life saved for every 114 screening CT scans performed, will transform the type of patients that we see to predominantly very early lung cancers $(1,2)$. Navigational Bronchoscopy will mean that someone (hopefully a surgeon) will go in bronchoscopically, take a biopsy and then ablate or freeze that nodule, and then sample all their N1 and $\mathrm{N} 2$ nodes at the same sitting. Then we will just follow the patient up, armed with their full list of available targeted therapies, should they relapse. Advanced Surgery will be reserved only for patients with areas of resistant mutations after multiple rounds of targeted therapy. (These operations will be highly complex as they will have dense adhesions as immunotherapy causes an intense inflammatory reaction around tumours).

Over the coming years, we must all be very much ready for constant seismic change in our specialty and be prepared to move with the times, adopt new technology fast, learn navigational bronchoscopy, understand the multiple targeted therapies, and learn new ways to operate on advanced cases. We will not be able to stay complacent or happy with our current 3-port VATS technique with open instruments, therefore, every surgeon needs to be watching for each latest development as it happens. Thus, I hope this has set the scene as to why it is so important to know what is on the horizon. Thus, I will share some of the latest developments that I have seen and encourage you to get on YouTube or start to ask about them and plan to evaluate some of the new technologies as they come out.

There are eight new platforms to look out for. The big companies are investing very heavily in high-quality robotic systems to rival Intuitive in the future. The Medtronic Robotic System is currently called 'Hugo' and is a neat plug and play design with independent arms on modules that can be wheeled to the patient and a surgeon console that can also be moved fairly freely. The major advantage that the Medtronic system will have over the competition is outstanding compatibility with its range of Covidien staplers and energy devices and the excellent network of support already available provided by Medtronic. Look out for working versions of this platform in 2019/20. Also, look out for a transformation in the business model. Medtronic will not come to you asking you to buy their robot. They will come to your hospital to renegotiate everything they sell to your hospital as a package. The stapling cost may rise a little, the energy device cost may rise, and they may ask your hospital to change some supplies from competitors to them, and the rewards will be the inclusion of a free robotic system. The costs will be packaged and leased with upgrades included and guarantees that you will not be left behind with 'last year's' model in the same way that you probably have your iPhone on a 2-3-year rolling contract.

The second giant is the Ethicon-Google pairup in the form of a company called VERB surgical (www.verbsurgical.com). Their selling model will be the same as Medtronic, with it packaged with all their other products, predicated on the guarantee of exclusivity and loyalty. But their entrance into is market is the most ambitious project of all of the start-up companies. With the energy of Google, and actually being developed in Google's original office buildings, there are visionary features being developed like intelligent machine learning, google hangouts, multiplatform sharing of videos in active development. The system itself is rumoured to have the arms coming out from under table and the company itself is describing it as being 'always there and always on' and being a whole new way of performing integrated surgery, bringing in scan data and perioperative data into one unified system. In addition to this, they might lead a revolution in how we plan for our operations, from the current situation... which is basically little or no planning, to taking the preoperative scans into simulation software, where the whole operation will be performed in a virtual operating room and also compared to videos of similar operations performed elsewhere in the world where software will identify the most similar cases for you to look at and learn from.

Sounds impossible? Well, Google have done it with Google-everything, from Google Earth, to Google Hangouts, to Google Translate, Google Classroom, etc. Together with total compatibility with everything that Ethicon have to offer, this will be an incredible platform of the future. Genius like this takes time, so 2020 or later may be the first time that you will be able to see it working on 
patients, but the cost is likely to be high in comparison to other models.

TransEnterics have a currently working robotic platform that is in clinical use called the Senhance surgical system (www.transenterix.com). They feature $5 \mathrm{~mm}$ instruments, each arm on a separate moveable gantry, haptic feedback and a camera controlled by your own head movement. However, they promote themselves as a cost-sensitive solution and therefore have made some compromises in the system, including many of the instruments not being wristed. In addition, the controllers look like laparoscopic instrument handles and thus many describe the system as a remote laparoscopic instrument holder, but with quite a large price. As a result, they are still loss-making and their website reports the sale of only four systems in the $2^{\text {nd }}$ quarter of 2018 at $\$ 1$ million each and they are losing an adjusted net loss of $\$ 11$ million per quarter as a company at the moment.

AvateraMedical (www.avatera.eu) are a German company who are developing a 4-arm robot from a single cart in a similar fashion to Intuitive with a closed surgeon console again similar to Intuitive. Not much is known about this system, other than it is very similar to the Intuitive system. And not to be outdone, there is another system called REVO-1 manufactured in South Korea that was launched for clinical use in 2018 (www.revosurgical.com). This takes the similarity of its system to the Intuitive system to a new dimension. It is a 4-arm, single cart system, with a very similar closed surgeon console with the main differentiation being price.

Medicaroid are a Japanese company (www.medicaroid. $\mathrm{com} /$ ) whose main interesting factor is that they have paired up with Kawasaki, the giant robotic manufacturer of car assembly plants. There are rumours online that it may have the arms in the table but I do not know much about this system and currently there is little known about developments of this system or release timings, so I do not think we will see anything until after 2021.

CMR Surgical is a UK company that have a working system called Versius and are ready to install this into six UK hospitals in the next 6-12 months, with CE marking expected in the first quarter of 2019 ( www.cmrsurgical. com.) My own hospital hopes to be one of these six. This was designed in reverse to usual systems as they asked the question as to what they thought the UK system could sustain financially for a robotic system. They came up with the answer that it could not sustain any upfront cost and the per-case costs had to be the same as current reusable laparoscopic instruments. Thus, they embarked on designing a system that did this. Two hundred Cambridge Graduate Engineers have now designed an immaculate system with $5 \mathrm{~mm}$ robotic arms, each standing on their own small portable modules to be wheeled up to the patient. It uses any standard endoscopic ports and has a surgeon's console with hand controls far more like an Xbox than a cardiac surgeons Castro needle holders. It is very small and portable, and interestingly you can raise the surgeon's console up to a standing height enabling you to be double gowned and gloved in case an immediate return to the table is needed, or if you would like to do the staple firing yourself.

But the final king of the future in thoracic surgery will surely be the Intuitive Da Vinci SP surgical System. This finally has FDA approval for urology and is now in clinical use in the USA and is the holy grail for thoracic surgery. With three robotic arms and an amazing snake camera all through a single $2.5 \mathrm{~cm}$ port that spread apart on entering the chest, finally uniportal robotics is here, which will not only make uniportal robotics far more simple for all users but will open up the reality of subxiphoid-only uniportal robotic surgery, which must surely be the least invasive approach in Thoracics possible. Currently, subxiphoid uniportal is performed in a very tiny minority of cases due to its extreme technical difficulty, but it will now be possible to do this for virtually every thoracic case, as long as you have around \$3-4 million to spare!

Finally, I would like to caution you against assuming that pure robotics is the only future. We will see a melding of VATS and Robotics with the advent of wristed VATS instruments. I have had the pleasure of using the FlexDex surgical instrument (www.flexdex.com) for lobectomy, thymectomy and diaphragm plication. Currently, version 1 is only a needle holder, but version 2 will have Maryland graspers with bipolar energy, Cadiere style graspers, hooks, scissors and maybe even suture-cut needle holders. At only a few hundred dollars each, this brings wristed instrumentation to all VATS Surgeons. They are FDA approved and CE marked.

FlexDex are not the only company working on wristed instrumentation. A company called LIVSMED (www. livsmed.com) from South Korea have been demonstrating a suite of fully wristed instruments that can be used in both hands that seem to be very similar to robotic marylands, graspers, hooks and needle holders. They tell me that they already have a full suite of instruments and have performed cases clinically in South Korea, although they are not 
FDA approved or CE marked. We hope to have a clinical trial of these instruments approved in the UK in the next six months.

Thus, one alternative glimpse of the future is to be having two wristed instruments in your hands and a robotic camera holder such as autolap or freehandsurgical (www. mst-sys.com or www.freehandsurgeon.com ) with a $3 \mathrm{D}$ Camera. With this set up, you have every element of a robotic system (i.e., wristed instrumentation, control of the camera, 3D vision) and none of the disadvantages (assistant required to perform the stapling, surgeon away from the patient's bedside) and because you do not need an assistant, this set up will actually be cheaper than the VATS surgery offered today, and safer than current robotics.

The future of thoracic surgery will certainly change quickly, so we must move with it. I have mentioned some of the novel robotic and wristed VATS instruments that will be available very soon but if I had one piece of advice for all surgeons, it would be to be looking at navigational bronchoscopic systems. The current available system is called Superdimension www.superdimension.com from Medtronic, but new entrants to the market are already coming, including the $\$ 700$ million company called Auris (www.aurishealth.com) who have developed a 'robotic' bronchoscope purely because they see the future of biopsy and ablation. The industry already sees this as a huge opportunity and with the advent of lung cancer screening, transformational change is sure to happen in bronchoscopy. If you don't believe me, then ask Intuitive. They have just bought Auris, even before it has fully entered the market, so sure are they that this is the future of our specialty.

I have also seen a 3-mm filament for a bronchoscope with $100 \times$ magnification allowing on-table, real-time microscopy in the lungs to identify tumour tissue as opposed to inflammation or normal alveoli. This has been developed in Edinburgh and is ready for clinical trials. These developments are just around the corner. All these systems require general anesthesia and we must learn the lessons of the cardiac surgeons who were slow to enter the catheter labs and who lost the leadership in TAVI and revascularization. We must enter the world of bronchoscopy and embrace this more minor procedure with as much enthusiasm as subxiphoid uniportal robotic surgery.

Finally, we have not even discussed the word 'robotic'. Ask the man in the street what robotics means (or even a patient referred for lobectomy) and they will tell you that it will be like the automated car production lines or driverless trains.
In fact, we do not have robotic surgery at all currently, and all the systems above are in fact like the first 'fly-bywire' planes when the physical connections from the pilot's control column to the plane's rudder and ailerons were replaced by computer interfaces and wires to aileron motors to allow smoother and more accurate movements. And just as with aviation, we are about to see a transformation in 'assisted' surgery. This does not mean that you will sit having a cup of tea or press a button and just watch it happen. Just like automated driving technology, it can mean many levels of assistance. If we consider the analogy of automated driving, you can have a range of assistance, from warnings of collisions and alarms, cruise control, and adaptive cruise control, to in-lane navigation where the car will steer on an easy motorway and keep you in lane, all the way to full autonomous driving.

So it will be in surgery. We will start with warning systems when the pulmonary artery looks like it is being stretched too much, or when it notices aberrant anatomy on the CT scan, or navigation systems begin to guide us to the tumour. We may then have pre-planned chest entry systems, that will place the ports in the correct intercostal space, and dock the arms, exchanging arms when required, and control $\mathrm{CO}_{2}$ pressures for us (I have seen one company's robotic arms docking with the ports themselves). And then later we will see increasing automation, with safe automated stapling, and then automated bronchial anastomosis for sleeve resections. This may come later, but the lesson of technology development in multiple industries is that it will come when it makes the procedure safer, quicker, cheaper or just a lot better.

This rapid change is however leaving our archaic surgical training model behind and our concept of a surgeon's 30year career pathway in tatters. We will need to innovate these pathways just as rapidly in order to embrace these changes and allow our patients to benefit from new technology, and for us to be the ones offering it to them as they become available.

\section{Acknowledgements}

None.

\section{Footnote}

Conflicts of Interest: The author has no conflicts of interest to declare. 


\section{References}

1. De Koning H, Van Der Aalst C, Ten Haaf K, et al. PL02.05 Effects of Volume CT Lung Cancer Screening: Mortality Results of the NELSON RandomisedControlled Population Based Trial. J Thorac Oncol

Cite this article as: Dunning J. Disruptive technology will transform what we think of as robotic surgery in under ten years. Ann Cardiothorac Surg 2019;8(2):274-278. doi: 10.21037/ acs.2019.03.02
2018;13:S185.

2. National Lung Screening Trial Research Team, Aberle DR, Adams AM, et al. Reduced lung-cancer mortality with low-dose computed tomographic screening. N Engl J Med 2011;365:395-409. 